\title{
Análisis crítico del currículo escolar en Chile en función de la justicia social
}

\author{
Critical Analysis of the School Curriculum in Chile Based on Social Justice
}

\section{Análises crítica do currículo escolar no Chile com base na justiça social}

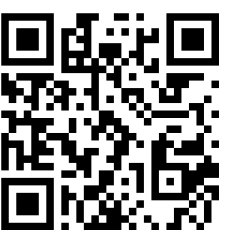

\author{
Felipe Nicolás Mujica-Johnson \\ Universidad Católica de Temuco \\ Centro de Investigación Escolar y Desarrollo \\ Temuco, Chile \\ fmujica@live.cl \\ (D) https://orcid.org/0000-0002-6956-2357
}

Recibido $\cdot$ Received $\cdot$ Recebido: 08 / 01 / 2018
Corregido $\cdot$ Revised $\cdot$ Revisado: 03 / 04 / 2019
Aceptado $\cdot$ Accepted $\cdot$ Aprovado: 13 / 11 / 2019

Resumen: La educación escolar en Chile se encuentra compuesta por un currículo que responde esencialmente a un enfoque positivista, denominado pedagogía por objetivos. Por consiguiente, el estudiantado es clasificado según su rendimiento académico y se le prepara para competir en diferentes pruebas estandarizadas, las cuales se encuentran condicionadas a estímulos económicos y a la oportunidad de continuar los estudios en la educación superior. Ante esta situación, el objetivo de este ensayo fue analizar en forma crítica los objetivos de aprendizaje y la evaluación del currículo escolar chileno en función de la perspectiva de la justicia social. En el inicio del análisis se describe la inadecuada estandarización del currículo, ya que los objetivos de aprendizaje se caracterizan por su ambigüedad y, en cuanto a las evaluaciones sumativas, no existen lineamientos ministeriales claros. Así, se argumenta que esto produce una competencia desigual entre el alumnado, el profesorado y los diferentes centros educativos. Sin embargo, se identifica que no es adecuado continuar estandarizando el currículo, más bien es necesario que la lógica del currículo escolar dé un giro epistemológico hacia una perspectiva constructivista y sociocrítica. De esta forma, se podrá progresar hacia una educación emancipadora que trabaje para la justicia social.

Palabras claves: Educación formal; docencia; aprendizaje; positivismo; evaluación del currículo; estandarización. 
doi: http://doi.org/10.15359/ree.24-1.25

URL: http://www.una.ac.cr/educare

CORREO: educare@una.cr

\begin{abstract}
School education in Chile is composed of a curriculum that essentially responds to a positivist approach, called pedagogy by objectives. Therefore, school children are classified according to their academic performance and are prepared to compete in different standardized tests, which are conditioned to economic stimuli and the opportunity to continue their studies in higher education. Given this situation, this essay aimed to critically analyze the learning objectives and the evaluation of the Chilean school curriculum in terms of the perspective of social justice. At the beginning of the analysis, the inadequate standardization of the curriculum is described since ambiguity characterizes the learning objectives, and in terms of summative evaluations, there are no clear ministerial guidelines. Thus, it is argued that this produces unequal competition among students, teachers, and different educational centers. However, it is identified that to continue standardizing the curriculum is not appropriate; instead, the school curriculum logic must make an epistemological turn towards a constructivist and sociocritical perspective. In this way, it will be possible to progress towards an emancipatory education that works for social justice.
\end{abstract}

Keywords: Formal education; teaching; learning; positivism; curriculum evaluation; standardization.

Resumo: A educação escolar no Chile está composta por um currículo que responde essencialmente a uma abordagem positivista, chamada pedagogia por objetivos. Portanto, o grupo de estudantes é classificado de acordo com seu desempenho acadêmico e é preparado para competir em diferentes provas padronizadas, condicionadas por estímulos econômicos e pela oportunidade de continuar os estudos no ensino superior. Diante dessa situação, o objetivo deste ensaio foi analisar criticamente os objetivos de aprendizagem e a avaliação do currículo escolar chileno com base na perspectiva da justiça social. A padronização inadequada do currículo é descrita no início da análise, uma vez que os objetivos de aprendizagem são caracterizados por sua ambiguidade e, no que diz respeito às avaliações sumativas, não há diretrizes ministeriais claras. Assim, argumenta-se que isso produz uma competição desigual entre estudantes, professores e diferentes centros educacionais. No entanto, identifica-se que não é apropriado continuar padronizando o currículo, mas é necessário que a lógica do currículo escolar dê uma volta epistemológica em direção a uma perspectiva construtivista e sócio-crítica. Dessa maneira, será possível progredir no sentido de uma educação emancipatória que trabalhe pela justiça social.

Palavras-chave: Educação formal; ensino; aprendizado, positivismo; avaliação curricular; padronização

\title{
Introducción
}

El currículo escolar en Chile responde a un modelo pedagógico por objetivos, que esencialmente responde a un enfoque positivista de la educación, de modo que en cada asignatura disciplinar el profesorado tiene un listado de objetivos de aprendizaje (OA, en adelante) que desarrollar con su alumnado y el resultado de este proceso será evaluado en forma sumativa, asignando una nota a cada estudiante. Para asegurar la calidad educacional a nivel nacional y ofrecer una base cultural común para todo el país, el Ministerio de Educación 
(MINEDUC, en adelante) ha establecido una reciente actualización de las bases curriculares de primero a sexto año básico en el decreto n. ${ }^{\circ}$ 439/2012 (Ministerio de Educación, 2011), de séptimo año básico a segundo año medio en los decretos n. ${ }^{\circ} 614 / 2013$ y n. ${ }^{\circ}$ 369/2015 (Ministerio de Educación, 2016a) en las que se encuentran los OA que deben desarrollar todos los establecimientos educacionales que cuenten con un nivel escolar en el país, pues aborda el contenido mínimo y obligatorio que cada institución debe trabajar. Además, el MINEDUC proporciona programas educativos para cada nivel escolar y asignatura, el cual representa una propuesta educativa, ya que los centros escolares tienen la posibilidad de desarrollar su propia propuesta de proyecto educativo, lo cual permite a los centros particulares llevar a la práctica su forma independiente de hacer pedagogía.

El problema que se abordará en este manuscrito se encuentra inmerso en el proceso descrito brevemente en el primer párrafo, debido a que los objetivos de aprendizaje no son del todo precisos, como se estableció en un análisis de contenido realizado a los programas y bases curriculares de primer a sexto año básico en la asignatura de educación física y salud, el cual concluye que "existe ambigüedad en los objetivos de aprendizaje de las bases curriculares y en los indicadores sugeridos en los programas de estudio" (Mujica y Concha, 2015, p. 24). En consecuencia, se ha creado un contexto pedagógico en que cada docente puede interpretar en forma diferente un $\mathrm{OA}$, el cual fue establecido a nivel nacional, porque define el aprendizaje necesario que el alumnado debe lograr para su formación. Esta flexibilidad en torno a un OA, permite que, en un mismo nivel educativo de diferentes centros escolares, se produzcan diferencias en el volumen de información o práctica desarrollada, y en la dificultad con la que se aborda el contenido del mismo OA.

El segundo problema que existe en relación con el desarrollo de las bases curriculares es que el MINEDUC no proporciona una evaluación sumativa de los OA, que tiene la función de "verificar el grado de alcance de los objetivos propuestos previamente" (Cejas y Álvarez, 2006, p. 150). Así, el profesorado debe construir sus propias evaluaciones, para un objetivo que se encuentra estandarizado a nivel nacional, lo cual se torna ilógico, ya que es imposible que no existan diferencias o errores en todas las evaluaciones creadas, que perjudiquen a algunos sujetos y beneficien a otros del sistema educativo. Esto se explica en la subjetividad que cada docente le otorga a su quehacer pedagógico. Esta subjetividad incluye razonamientos, creencias e ideologías que también se integran a la afectividad del profesorado (Mujica, 2018). Aquel tema suele ser considerado en las investigaciones, como las teorías implícitas o subjetivas del profesorado en torno a múltiples otras dimensiones, incluyendo también la vocación docente como factor importante en las decisiones y la resiliencia del profesorado (Gu y Day, 2013; Mujica y Orellana, 2018). De esta forma, el estudio realizado por Robles, Giménez y Abad (2010), que contó con la participación de un grupo de profesorado de educación secundaria de España, logró identificar que diferentes decisiones curriculares son tomadas en función de la interpretación 
doi: http://doi.org/10.15359/ree.24-1.25

URL: http://www.una.ac.cr/educare

CORREO: educare@una.cr

del contexto pedagógico, al considerar factores psicológicos, materiales, socioculturales. Pero, además, un factor importante era la experiencia y el conocimiento de cada docente. Cabe destacar que para comprender las teorías implícitas del profesorado es preciso considerar la formación escolar y la formación inicial docente, ya que un estudio realizado con estudiantes de pedagogía en la zona sur de Chile identifica diferencias sobre la comprensión textual y la competencia lectora, destacando también los modelos culturales como factores que pueden haber incidido (Makuc, 2011).

En este sentido, el rol que asume actualmente el MINEDUC, en esta fase del proceso educativo, se limita a sugerir indicadores de evaluación, por medio de los programas escolares, los cuales no es obligatorio aplicarlos. Sumado al problema de la falta de delimitación en la definición de los objetivos, el profesorado tiene que lidiar con toda la responsabilidad del proceso evaluativo. La lógica que aplica el MINEDUC es considerar que todo el profesorado es especialista en la construcción de instrumentos de evaluación sumativa, los cuales, además, serán tan bien construidos, que contarán con una excelente validez, confiabilidad y practicidad (Foronda y Foronda, 2007). De esta manera, se podría evidenciar en forma exacta el resultado de aprendizaje del alumnado, lo cual se expresa en la siguiente cita:

Los profesores deben tener muy claro cómo se evaluará el trabajo de los estudiantes, especialmente porque frente a aprendizajes más complejos donde posiblemente no se encuentre sólo una respuesta correcta, se requiere identificar y comunicar muy claramente cuáles son los criterios de evaluación que se utilizarán, ya que éstos determinan el tipo de "producto" que es esperado y cuáles son los aspectos centrales que determinarán que los estudiantes aprendieron.

Finalmente, resulta relevante que estos criterios sean coherentes con las metas propuestas por el currículum nacional de manera asegurar que los aprendizajes de los estudiantes sean equitativos y comparables a los estudiantes de otros establecimientos del país. (Ministerio de Educación, 2008, p. 21)

Esta lógica se aprecia bastante lejana a la realidad, ya que, hasta la actualidad, no existe un currículo estandarizado en la formación de docentes en Chile, más bien existe una evidente desregulación del sistema de educación superior, por lo que se están tomando medidas para unificar criterios (Ruffinelli, 2013), sumado a que construir un instrumento de evaluación fiable es una tarea que demanda bastante tiempo y complejidad (Cejas y Álvarez, 2006). Por consiguiente, en la actualidad la evaluación es un tema bastante polémico y discutido, ya que es difícil llevarla a la práctica, por la tendencia a reducirla a niveles meramente instrumentales (Ávila y Paredes, 2015). 
Además de los problemas mencionados que presenta el currículo por objetivos en Chile, también existiría otro problema más de fondo de carácter epistemológico, debido a que la lógica vigente desconoce, en gran medida, la realidad histórico-cultural de las comunidades educativas, que incluyen a familias, alumnado, profesorado y autoridades de los centros educativos. De esta forma, se produce una competencia muy desigual entre el alumnado, el profesorado y también el personal directivo de los diferentes centros educativos, bajo estándares que privilegian a los sectores más favorecidos del país, principalmente en términos culturales y socioeconómicos. Pero, ¿por qué una competencia? Principalmente, porque los resultados de los aprendizajes son calificados en datos numéricos o notas, que posteriormente incidirán en posteriores beneficios económicos estatales tanto para el alumnado, el profesorado y los centros educativos. Además, con base en aquel rendimiento escolar variarán las posibilidades del alumnado para poder estudiar alguna carrera universitaria, una vez finalizada la educación escolar.

Sobre el acceso a la educación superior en Chile, Espinoza y González (2015) indican que el mayor porcentaje de estudiantes que ingresa a la universidad y proviene de centros educativos científicos-humanistas pertenece a la clase más rica del país. Por lo tanto, para el alumnado de los sectores menos favorecidos "se genera una doble discriminación que se debe a dos razones: por una parte, su origen socioeconómico y, por otra, la inadecuada preparación que reciben durante su paso por la enseñanza media" (p. 522). Cabe destacar, también, que el problema de la segregación escolar y la desigualdad educativa en Chile ha sido ampliamente estudiado, identificándose como uno de los principales factores que han contribuido a esta situación, las políticas neoliberales que aumentaron los niveles de precariedad social y perjudicaron la educación pública del país (García-Huidobro, 2007; Oliva, 2008; Oliva y Gascón, 2016; Puga, 2011 ). En consecuencia, es coherente que para enfrentar esta realidad se propicien modificaciones, en la educación, orientadas hacia la consecución de justicia social.

Sobre una educación basada en la justicia, es pertinente considerar los planteamientos de Murillo y Hernández-Castilla (2014), quienes señalan lo siguiente:

Una escuela que trabaja para la Justicia Social debe ser una escuela que aborda la Justicia Social en los procesos de enseñanza, en sus objetivos, contenidos, metodologías y estrategias de evaluación; pero también tiene que ser una escuela justa, que funciona y se organiza bajo los planteamientos de la Justicia Social, con una cultura escolar cuyo máximo valor es hacer una escuela de todos y para todos y donde no quede rastro de discriminación o exclusión. (p. 29)

A partir de las problemáticas mencionadas, se ha elaborado este ensayo pedagógico que pretende analizar desde una perspectiva crítica los objetivos de aprendizaje y la evaluación en el currículo escolar chileno en función de la perspectiva de la justicia social. Por lo tanto, el análisis también discute la lógica que sostienen aquellos componentes curriculares. 
doi: http://doi.org/10.15359/ree.24-1.25

URL: http://www.una.ac.cr/educare

CORREO: educare@una.cr

\section{Currículo escolar en Chile}

La pedagogía por objetivos, todavía vigente en el currículo escolar de Chile, surge del paradigma positivista, en respuesta a una visión racionalista de la educación, el cual reduce el conocimiento a una lista de objetivos empíricamente observables (Álvarez, 2001), el cual hunde sus raíces en el movimiento utilitarista en educación que se desarrolló en Estados Unidos, paralelo al auge que el enfoque taylorista tuvo en la industria (Gimeno, 1986). Desde esta lógica educativa, un OA representa una formulación didáctica que expresa en forma clara y precisa los cambios de conducta en el área cognitiva, afectiva o psicomotriz, "que se han de operar en el alumno como efecto del proceso enseñanza-aprendizaje" (Salcedo, 2011, p. 116).

Por lo tanto, si en Chile todavía se adopta un modelo pedagógico positivista, tendría que poder responder a las exigencias que se encuentran implícitas, ya que "los objetivos se desagregan según grados progresivos de especificación y concreción, conformando un cuerpo coherente en que cada nivel de concreción mayor, apunta a logros intermedios, en realidades más acotadas y en plazos menores con respecto a los anteriores" (Batistón y Ferreyra, 2005, p. 95). Al revisar el discurso ministerial, se aprecia un convencimiento de que existe una adecuada estandarización del currículo escolar, señala que las bases curriculares definen específicamente los desempeños mínimos que se espera que todo el estudiantado logre en cada asignatura y nivel de enseñanza (Ministerio de Educación, 2013). Sin embargo, es una afirmación que se ha demostrado no es exacta (Mujica y Concha, 2015), de modo que especialistas de cada disciplina tienen la tarea de analizar cómo mejorar el contenido de su currículo. Para ejemplificar la problemática mencionada, se ha tomado el OA 13, correspondiente a la unidad 4, del curso primer año medio, de la asignatura Lengua y Literatura, que dice lo siguiente:

Escribir, con el propósito de explicar un tema, textos de diversos géneros (por ejemplo, artículos, informes, reportajes, etc.) caracterizados por:

> Una presentación clara ... que se esbozan los aspectos que se abordarán.

$>$ Una organización y redacción propias de la información.

$>$ La inclusión de hechos, descripciones, ejemplos o explicaciones que reflejen una reflexión personal sobre el tema.

$>$ Una progresión temática clara, con especial atención al empleo de recursos anafóricos y conectores.

$>$ El uso de imágenes u otros recursos gráficos pertinentes.

$>$ Un cierre coherente con las características del género y el propósito del autor.

$>$ El uso de citas y referencias según un formato previamente acordado. (Ministerio de Educación, 2016b, p. 30) 
En aquel objetivo, se puede apreciar la falta de delimitación en el "etc.", que deja la posibilidad a incluir una diversidad de textos, lo cual no es un problema en la riqueza de contenidos, sino que el problema estaría en que mientras en un centro escolar se estudiaron cuatro diferentes textos, en otro puede que solamente se haya estudiado el reportaje, ya que no se explicita la cantidad de textos que se deben abordar. Así, se convierte en un enunciado con un complejo contenido utilitario y sistematizador (Salcedo, 2011). En él también se señala una presentación y progresión "clara" o un cierre "coherente", pero no se especifica la operacionalización de esas categorías, generando la posibilidad de que un grupo de docentes las interpreten con exigencias diferentes. Mencionando solo esas minúsculas ambigüedades, se comienzan a crear diferencias en el volumen de información estudiada y en la dificultad que establece cada docente, pues se genera una diversidad de criterios que resta validez y consistencia al proceso docente y sus resultados (Salcedo, 2011). Por ende, ante este OA, caben las siguientes interrogantes: ¿hay diferencias de aprendizaje entre un curso que se les ha exigido escribir cuatro textos, versus, otro que se les exigió escribir siete? En cuanto a la evaluación sumativa del objetivo, ¿obtendrían puntajes diferentes estudiantes que hayan colocado nueve imágenes versus estudiantes que colocaron tres? ¿En una rúbrica de evaluación, asignaría el profesorado el mismo puntaje a los diferentes criterios establecidos para el aprendizaje? Estas preguntas y observaciones demuestran lo frágil que es la objetividad en el desarrollo de un objetivo que no se encuentra bien delimitado, además de una ausencia de unificación sobre los mejores criterios pedagógicos para afrontar las evaluaciones estandarizadas. Así mismo, esta situación es propicia para que algunos centros educativos aprovechen para sobresalir o triunfar en los resultados académicos del alumnado de las diferentes pruebas estandarizadas que se realizan a nivel nacional. De esta forma, lograrían obtener un mayor prestigio en la comunidad y una mejor captación del alumnado.

Ante esta situación, si las políticas públicas del país sostienen un currículo pedagógico estandarizado, basado en la competitividad escolar, debe considerar que una gran cantidad de objetivos tienen que ser analizados y delimitados, así como el profesorado tiene que ser apoyado en la tarea de evaluar, lo cual representa un enorme desafío, ya que solo en las bases curriculares de primero a sexto año básico existen 791 OA entre todas las asignaturas. No obstante, se considera que las autoridades educativas del país deben trabajar en una dirección que no sea la de continuar estandarizando el currículo escolar y se sumen a las iniciativas curriculares constructivistas y sociocríticas para transformar la lógica del sistema educativo, partiendo de la idea de que todo proceso educativo es único, porque responde a un contexto histórico-cultural concreto (Vygotsky, 1984). Por lo mismo, el profesorado debe reaccionar en forma crítica a la labor que está cumpliendo para detectar las discriminaciones que se reproducen en el sistema educativo, aportando a la justicia social y a la igualdad de oportunidades.

Esta transformación curricular no se contradice con que los planes de estudios deben tener una sustentación teórica coherente entre objetivos, contenidos y el proceso de evaluación (Castillo-Cedeño, 2015), para de esta forma garantizar una educación de calidad para todas 
doi: http://doi.org/10.15359/ree.24-1.25

URL: http://www.una.ac.cr/educare

CORREO: educare@una.cr

las personas, que favorezca y certifique el derecho a aprender sin presiones institucionales innecesarias y absurdas (Sanz y Serrano, 2016).

Cabe destacar, que esta lógica positivista de estandarizar el currículo escolar, para comparar y equiparar resultados de aprendizaje, limita las posibilidades que tiene el profesorado para aplicar evaluaciones auténticas desde una perspectiva constructivista, en la que se hable de aprendizaje activo y situado (Álvarez, 2005). Sin duda que esta lógica positivista no responde a una perspectiva educativa del siglo XXI, lo cual se aprecia en la aceptación que ha tenido en el mundo la pedagogía por competencias (Ávila y Paredes, 2015).

Justamente, el enfoque pedagógico de las competencias se sustenta en los aportes que han logrado importantes especialistas que se distanciaron del enfoque conductista o positivista, para hacer del acto educativo un proceso más transversal, activo e integral. Entre ellos destaca Vygotsky (1984), que tras sus investigaciones logró identificar que "el aprendizaje es un momento intrínsecamente necesario y universal para que se desarrollen en el niño esas características humanas no naturales, sino formadas históricamente" (p. 115). A partir de aquel planteamiento, se deduce que el contexto histórico-cultural del alumnado, tanto a nivel familiar, social y escolar, afecta su aprendizaje para bien o para mal. En este sentido, lo más sensato es esperar que en todos los centros educativos existan diferentes realidades socioculturales $y$, por lo tanto, que también existan logros de aprendizaje adaptados a cada contexto pedagógico. Desde esta lógica orientada más hacia el constructivismo, toma sentido incorporar evaluaciones auténticas, así como autoevaluaciones, co-evaluaciones y evaluaciones compartidas que sean consideradas evaluaciones sumativas para verificar el desempeño o rendimiento del alumnado.

Este cambio de paradigma ha comenzado a concretarse en la educación superior y la formación inicial docente en Chile, pero como toda modificación, requerirá lidiar con la resistencia del profesorado y del alumnado que se encuentra más adaptado al paradigma tradicional. Asimismo, lo demuestra un estudio que indagó los procesos evaluativos en una universidad chilena, el cual concluye que a pesar de que se están aplicando evaluaciones formativas y compartidas, se aprecia "un predominio en el uso de instrumentos que no involucran al alumnado en la evaluación" (Gallardo-Fuentes, López-Pastor y Carter-Thuillier, 2017, p. 235). Esta situación además exige que se abra una línea de investigación, sobre cómo están evaluando los diferentes cuerpos docentes del país, que reciben muy poca orientación sobre esta fase del proceso enseñanza-aprendizaje.

\section{Transformar la lógica del currículo escolar chileno}

Como se ha mencionado anteriormente, para mejorar los problemas del actual enfoque tecnocrático por objetivos, la mejor opción no es seguir aumentando la estandarización del currículo, con la finalidad de aumentar la rigurosidad en el aprendizaje escolar. Si bien el 
aumento de la estandarización curricular es consecuente con las pruebas estandarizadas a la que es sometido el alumnado para poder continuar sus estudios en educación superior, se debe considerar la voz de especialistas que critican este modelo por su perspectiva autoritaria y rígida, que promueve una comunicación unidireccional del docente al estudiante, así como la reproducción memorística (Soubal, 2008), para poder responder con éxito a los exámenes con preguntas cerradas. En resumen, un modelo alejado del complejo sistema social de la actualidad, que requiere competencias muy diferentes a las de hace algunas décadas para propiciar la inserción de las personas en el ámbito social.

Como respuesta a este enfoque curricular positivista, que se ha planteado hace décadas como el único válido para la educación escolar y el paso a la educación superior, surge una campaña social Ilamada alto al SIMCE (Sistema de medición de la calidad educativa), la cual expresa el daño social y emocional que produce esta prueba estandarizada en el profesorado. Daño que principalmente se encuentra asociado a la relación de la remuneración socioeconómica del personal docente con el rendimiento escolar del alumnado, de modo que las consecuencias de aquella prueba se pueden transformar en una recompensa económica o en un castigo social (Campos-Martínez y Guerrero, 2016). Pensar que una definición más precisa de los OA y la aplicación de evaluaciones más estandarizadas disminuirá las injusticias sociales es un absurdo, ya que por ningún motivo son los factores que cambiarían drásticamente el panorama de la calidad y la segregación de la educación en Chile. Sobre todo, porque el acto educativo es un proceso multidimensional, dinámico y complejo que, ante todo, debe trabajar para "el desarrollo integral de niños, niñas, adolescentes y personas adultas, con el principio de apoyo diferencial a quien más lo necesita" (Belavi y Murillo, 2016, p. 31).

Sin embargo, el profesorado que se encuentra desempeñando su labor en los centros educativos no es el responsable de que en el país se mantenga vigente un modelo positivista, por lo que es injusto que sufran las consecuencias de un paradigma que les exige negar las particularidades de cada contexto educativo y competir en desigualdades condiciones con sus pares. Por estas razones, es fundamental que el MINEDUC asuma el desafío de transformar epistemológicamente el currículo escolar, considerando su rol como principal organismo que gestiona la educación en Chile.

Para que esta transformación sea exitosa, es fundamental la voluntad política, que se vislumbra como una de las principales barreras para avanzar hacia un paradigma más crítico de la educación chilena, sobre todo si se considera que en los países democráticos hay colectividades que se benefician de las injusticias sociales. Por lo mismo, para enfrentar esta situación, Belavi y Murillo (2016) sostienen que la transformación hacia una educación basada en la justicia social debe comenzar en la misma educación, por medio de una pedagogía emancipadora que oriente la moral del alumnado y que le permita participar activamente de la vida social y democrática del país. 
doi: http://doi.org/10.15359/ree.24-1.25

URL: http://www.una.ac.cr/educare

CORREO: educare@una.cr

Para comprender la dificultad que significa avanzar hacia cambios de fondo del sistema educativo escolar chileno, basta señalar que la desigualdad de la educación en Chile fue diseñada políticamente (Cavieres, 2014; Contreras y Macías, 2002; García-Huidobro, 2007; Oliva, 2008; Puga, 2011), por medio de políticas educativas de privatización que han conducido a una grave segregación escolar. Asimismo, su currículo ha sido parte de un acto de significación ideológica neoliberal; legitima estructuras sociales, económicas, políticas y culturales hegemónicas, además de las desigualdades materiales y simbólicas que las constituyen (Plá, 2016). Además, reiteradamente se han realizado críticas al currículo escolar chileno sobre el carácter reproductivo de los aprendizajes y la perspectiva tecnocrática dominante de estandarización (Donoso, 2005; Moreno, Gamboa y Poblete, 2014; Moreno, Rivera y Trigueros, 2014; Oliva y Gascón, 2016). Principalmente, porque ha convertido el acto de educar en un proceso rígido, con una estructura lineal y vertical, donde la docencia debe procurar no salirse de los márgenes establecidos, en una lógica bancaria de la educación (Freire, 1978).

Justamente, Murillo, Roman y Hernández (2011) plantean que para avanzar hacia una lógica educativa diferente, basada en la justicia social, se requiere dar un giro o cambio de sentido que afecte la esencia del enfoque predominante o mercantil de la educación, debido a que esta mirada "exige superar su actual condición de ser un servicio ofrecido, orientado y regulado desde criterios y principios de mercado, para pasar a aprehenderse y levantarse como un derecho ejercido en plenitud por todo niño, niña o joven en formación" (p. 11). Considerando aquellas orientaciones, es posible identificar dos caminos para transformar la lógica curricular en Chile, pero es preciso señalar que ambos caminos no se excluyen el uno al otro. El primero tendría una dirección de arriba hacia abajo o, en otras palabras, de la autoridad educativa a las bases educativas. Sería el camino más corto y en concreto, consistiría en que el MINEDUC, organismo liderado por el gobierno de turno, atienda el llamado de personal experto en educación y demuestre voluntad política para establecer el giro epistemológico del currículo. Mientras que el segundo camino tendría una dirección contraria, de las bases educativas hacia el MINEDUC, de modo que sería el más largo. En concreto, consistiría en fomentar por diferentes vías la discusión y conciencia moral en la sociedad, sobre la importancia de adoptar una lógica curricular que contribuya a la justicia social. Así, se propiciaría un diálogo entre la ciudadanía, el profesorado de los centros educativos, el profesorado de las universidades y las autoridades educativas, que permita lograr importantes acuerdos sobre la temática.

Cabe mencionar que ambos caminos para transformar esencialmente la lógica positivista en la educación chilena han comenzado en las universidades del país, al adoptar la lógica de la educación por competencias, y se espera que pronto alcancen al sistema educativo escolar, por lo que es fundamental que la comunidad educativa esté bien preparada para seguir participando activamente de aquel proceso. En este sentido, se ha de tener en cuenta de que para saber si un sistema educativo contribuye a la justicia social, debe ser evaluado en su "capacidad para igualar oportunidades, procesos y resultados y ser un motor de movilidad social para todos" (Murillo et al., 2011, p. 17). 


\section{Consideraciones finales}

El análisis realizado en torno al currículo escolar chileno puede resumirse en tres principales conclusiones de relevancia para una educación escolar que pretenda disminuir la brecha de la calidad y la segregación educativa, aportando de esta manera a la justicia social en el país.

En primer lugar, ante el modelo tecnocrático basado en la pedagogía por objetivos, la ausencia de lineamientos ministeriales precisos en el currículo escolar genera una competencia desigual entre los diferentes centros educativos, que afecta el proceso completo de escolarización y, en consecuencia, las oportunidades del alumnado para continuar su formación en la educación superior.

En segundo lugar, la responsabilidad de evaluar un currículo de corte estandarizado que el Ministerio ha transferido al profesorado, aumenta la presión laboral y propicia una competitividad desigual entre el alumnado, el profesorado y los centros educativos.

En tercer lugar, se identifica la necesidad que tiene el currículo escolar chileno de dar un giro epistemológico hacia una perspectiva constructivista y sociocrítica, que revindique la importancia del aprendizaje activo y situado en un contexto histórico-cultural concreto. Del mismo modo, que permita integrar, con una amplia presencia, diferentes instrumentos de evaluación auténtica, sin la necesidad de tener que responder a exigencias que limiten la creatividad del profesorado. Para esto último es imprescindible que se cuestione el actual sistema de ingreso a la educación superior y se piense en una alternativa que no exija la estandarización del currículo, sobre todo ante la discriminación que se genera por la segregación escolar.

Finalmente, queda mencionar que este tema debe ser sometido a una amplia discusión, por lo que se hace necesario que aumenten las reflexiones académicas en torno a la problemática mencionada a nivel nacional e internacional.

\section{Referencias}

Álvarez, J. M. (2001). Evaluar para conocer, examinar para excluir. Madrid: Morata.

Álvarez, I. (2005). Evaluación como situación de aprendizaje o evaluación auténtica. Perspectiva Educacional, 45(1), 45-67. Recuperado de https://www.redalyc.org/ pdf/3333/333329100004.pdf

Ávila, M. y Paredes, I. (2015). La evaluación del aprendizaje en el marco del currículo por competencias. Omnia, 21(1), 52-65. Recuperado de http://132.248.9.34/hevila/ OmniaMaracaibo/2015/vol21/no1/4.pdf

Batistón, V. y Ferreyra, H. (2005). Plan educativo institucional. Buenos Aires: Ediciones Novedades Educativas. 
doi: http://doi.org/10.15359/ree.24-1.25

URL: http://www.una.ac.cr/educare

CORREO: educare@una.cr

Belavi, G. y Murillo, F. J. (2016). Educación, democracia y justicia Social. Revista Internacional para la Justicia Social, 5(1), 13-34. doi: https://doi.org/10.15336/riejs2016.5.1

Campos-Martínez, J. y Guerrero, P. (2016). Efectos indeseados de la medición de la calidad educativa en Chile. La respuesta de la sociedad civil. Cadernos Cedes, 36(100), 355-374. doi: http://dx.doi.org/10.1590/cc0101-32622016171351

Castillo-Cedeño,I.(2015). Educaren la justicia social por ella y paraesta:Unaluchaineludiblemente ética. Revista Electrónica Educare, 19(2), 467-478. doi: https://doi.org/10.15359/ree.19-2.24

Cavieres, E. (2014). La calidad de la educación como parte del problema. Educación escolar y desigualdad en Chile. Revista Brasileira de Educaçao, 19(59), 1033-1051. doi: http://dx.doi. org/10.1590/S1413-24782014000900011

Cejas, C. y Álvarez, P. (2006). Evaluación de los resultados del aprendizaje. Revista Argentina de Radiología, 70(2), 149-155. Recuperado de https://www.redalyc.org/ html/3825/382538441009/index.html

Contreras, D. y Macías, V. (2002). Desigualdad educacional en Chile: Geografía y dependencia. Cuadernos de economía, 39(118), 395-421. doi: http://dx.doi.org/10.4067/S0717$\underline{68212002011800005}$

Donoso, S. (2005). Reforma y política educacional en Chile 1990-2004: El neoliberalismo en crisis. Estudios Pedagógicos, 31(1), 113-135. doi: https://doi.org/10.4067/S0718$\underline{07052005000100007}$

Espinoza, O. y González, L. E. (2015). Equidad en el sistema de educación superior de Chile: Acceso, permanencia, desempeño y resultados. En A. Bernasconi (Ed.), La educación superior de Chile: Transformación, desarrollo y crisis (pp. 517-579). Santiago: Universidad Católica de Chile.

Foronda, J. M. y Foronda, C. L. (2007). La evaluación en el proceso de aprendizaje. Perspectivas, 19, 15-30. Recuperado de https://www.redalyc.org/articulo.oa?id=425942453003

Freire, P. (1978). Pedagogía del oprimido. Madrid: Siglo XX1.

Gallardo-Fuentes, F. J., López-Pastor, V. y Carter-Thuillier, B. (2017). ¿Hay evaluación formativa y compartida en la formación inicial del profesorado en Chile? Percepción de alumnado, profesorado y egresados de una universidad. Psychology, Society, \& Education, 9(2), 227238. doi: https://doi.org/10.25115/psye.v9i2.699

García-Huidobro, J. E. (2007). Desigualdad educativa y segmentación del sistema escolar. Consideraciones a partir del caso chileno. Revista Pensamiento Educativo, 40(1), 65-85.

Gimeno, J. (1986). La pedagogía por objetivos: Obsesión por la eficiencia. Madrid: Morata. 
Gu, Q.y Day, C. (2013). Challenges to teacher resilience:Conditions count. BERJ.British Educational Research Journal, 39(1), 22-44. doi: https://doi.org/10.1080/01411926.2011.623152

Makuc, M. (2011). Teorías implícitas sobre comprensión textual y la competencia lectora de estudiantes de primer año de la Universidad de Magallanes. Estudios Pedagógicos, 37(1), 237-254. doi: https://doi.org/10.4067/S0718-07052011000100013

Ministerio de Educación. (2008). Marco para la buena enseñanza. Santiago: República de Chile. Recuperado de https://www.docentemas.cl/docs/MBE2008.pdf

Ministerio de Educación. (2011). Bases curriculares educación básica. Santiago: Autor. Recuperado de https://educrea.cl/bases-curriculares-educacion-basica/

Ministerio de Educación. (2013). Educación física y salud. Programa de estudio. Primer año básico. Santiago: Autor. Recuperado de https://educrea.cl/wp-content/uploads/2015/11/ programa-de-estudio-1-basico-educacion-fisica-191115.pdf

Ministerio de Educación. (2016a). Bases curriculares. 70 básico a $2^{\circ}$ medio. Santiago: Autor. Recuperado de https://media.mineduc.cl/wp-content/uploads/sites/28/2017/07/BasesCurriculares-7\%C2\%BA-b\%C3\%A1 sico-a-2\%C2\%BA-medio.pdf

Ministerio de Educación. (2016b). Lengua y literatura. Programa de estudio. Primero medio. Santiago: Autor. Recuperado de https://www.curriculumnacional.cl/614/articles-34377 programa.pdf

Moreno, A., Rivera, E.yTrigueros, C. (2014). Sistema de medición de la calidad de la educación física chilena: Un análisis crítico. Movimento, 20(1), 145-167. doi: https://doi.org/10.22456/1982$\underline{8918.41485}$

Mujica, F. N. (2018). Educar y suscitar emociones en la educación: Análisis crítico de su contribución al desarrollo moral. Ensayos, 33(2), 15-27. Recuperado de https://revista. uclm.es/index.php/ensayos/article/view/1540/pdf

Mujica, F. N. y Concha, R. (2015). Objetivos de aprendizaje de primer a sexto año en educación física y salud: Análisis crítico de la documentación ministerial. Revista Motricidad Humana, 16(1), 18-25. Recuperado de http://www.revistamotricidad.com/wp-content/ uploads/2015/09/18-25-Mujica-Johnson-Felipe.pdf

Mujica, F. N. y Orellana, N. del C. (2018). Autopercepción de la vocación en docentes de educación física escolar en Chile. CPU-e. Revista de Investigación Educativa, 27, 203-229. doi: https:// doi.org/10.25009/cpue.v0i27.2563

Murillo, F. J. y Hernández-Castilla, R. (2014). Liderando escuelas justas para la justicia social. Revista InternacionaldeEducación paralaJusticiaSocial,3(2), 13-32. Recuperadodehttps://repositorio. uam.es/bitstream/handle/10486/666733/RIEJS 3 2 2.pdf?sequence=1\&isAllowed=y 
doi: http://doi.org/10.15359/ree.24-1.25

URL: http://www.una.ac.cr/educare

CORREO: educare@una.cr

Murillo, F. J., Román, M. y Hernández, R. (2011). Evaluación educativa para la justicia social. Revista Iberoamericana de Evaluación Educativa, 4(1), 8-23. Recuperado de https://revistas. uam.es/index.php/riee/article/view/4467

Oliva, M. A. (2008). Política educativa y profundización de la desigualdad en Chile. Estudios Pedagógicos, 34(2), 207-226. doi: http://dx.doi.org/10.4067/S0718-07052008000200013

Oliva, M. A. y Gascón, F. (2016). Estandarización y racionalidad política neoliberal: Bases curriculares de Chile. Cadernos Cedes, 36(100), 301-318. doi: http://dx.doi.org/10.1590/ cc0101-32622016171349

Plá, S. (2016). Currículo, historia y justicia social. Estudio comparativo en América Latina. Revista Colombiana de Educación, 71, 53-77. doi: https://doi.org/10.17227/01203916.71rce53.77

Puga, I. (2011). Escuela y estratificación social en Chile: ¿Cuál es el rol de la municipalización y la educación particular subvencionada en la reproducción de la desigualdad social? Estudios Pedagógicos, 37(2), 213-232. doi: http://dx.doi.org/10.4067/S0718-07052011000200013

Robles, J. Giménez, F. J. y Abad, M. T. (2010). Motivos que llevan a los profesores de Educación Física a elegir los contenidos deportivos en la E.S.O. Retos. Nuevas Tendencias en Educación Física, Deporte y Recreación, 18, 5-8. Recuperado de https://dialnet.unirioja.es/servlet/ articulo? codigo $=5410055$

Ruffinelli, A. (2013). La calidad de la formación inicial docente en Chile: La perspectiva de los profesores principiantes. Calidad en la Educación, 39, 118-154. doi: http://dx.doi. org/10.4067/S0718-45652013000200005

Salcedo, H. (2011). Los objetivos y su importancia para el proceso de enseñanza-aprendizaje. Revista de Pedagogía, 32(91), 113-130. Recuperado de https://www.redalyc.org/articulo. oa?id=65926549007

Sanz, J. R. y Serrano, Á. (2016). El desarrollo de capacidades en la educación. Una cuestión de justicia social. Sinéctica, Revista Electrónica de Educación, 46, 1-16. Recuperado de http:// www.scielo.org.mx/pdf/sine/n46/1665-109X-sine-46-00004.pdf

Soubal, S. (2008). La gestión del aprendizaje. Algunas preguntas y respuestas sobre en relación con el desarrollo del pensamiento en los estudiantes. Polis. Revista de la Universidad Bolivariana, 21, 311-337. Recuperado de https://journals.openedition.org/polis/2955

Vygotsky, L. S. (1984). Aprendizaje y desarrollo intelectual en la edad escolar. Infancia y Aprendizaje, 7(27-28), 105-116. doi: https://doi.org/10.1080/02103702.1984.10822045 